\title{
Erratum to: Asset price bubbles from heterogeneous beliefs about mean reversion rates
}

\author{
Xi Chen · Robert V. Kohn
}

Received: 24 August 2011 / Accepted: 23 May 2012 / Published online: 10 July 2012

(C) Springer-Verlag 2012

\section{Erratum to: Finance Stoch (2011) 15:221-241 DOI 10.1007/s00780-010-0124-x}

Theorem 5.1 of [1] draws correct conclusions, however the proof is incomplete. Indeed, the final paragraph appeals to a "basic comparison theorem for viscosity superand subsolutions, see e.g. Theorem 5.1 of [2]." Alas, the cited result from [2] concerns equations of the form $u(x)+F\left(D u, D^{2} u\right)-f(x)=0$, whereas the equation under consideration in [1] does not have this form.

The purpose of that final paragraph was to conclude that $\Phi(D) \leq P_{*}(D)$, where $P_{*}$ is the minimal equilibrium price and $\Phi$ is the unique $C^{2}$ solution of

$$
-\max \left\{\kappa_{1}(\theta-D), \kappa_{2}(\theta-D)\right\} \Phi^{\prime}-\frac{1}{2} \sigma^{2} \Phi^{\prime \prime}+\lambda \Phi-D=0
$$

with linear growth at infinity. Actually, appeal to a general comparison result is unnecessary. The desired conclusion follows easily from the fact that $P_{*}(D)$ is a viscosity supersolution, using the asymptotic properties of $P_{*}(D)$ and $\Phi(D)$ as $|D| \rightarrow \infty$. Thus Theorem 5.1 of [1] can be replaced with the following:

This research was partially supported by NSF grant DMS-0807347.

The online version of the original article can be found under doi:10.1007/s00780-010-0124-x.

X. Chen · R.V. Kohn $(\bowtie)$

Courant Institute of Mathematical Sciences, New York University, New York, USA

e-mail: kohn@cims.nyu.edu

Present address:

X. Chen

Royal Bank of Canada, Toronto, Ontario, Canada

e-mail: roger.chen@ rbccm.com 
Theorem 5.1 The equilibrium price $\Phi(D)$ identified in Sect. 4 and the minimal equilibrium price $P_{*}(D)$ discussed in Sect. 3 have the following properties:

(i) $P_{*}(D) \leq \Phi(D)$, and $\Phi(D)-P_{*}(D) \rightarrow 0$ as $|D| \rightarrow \infty$.

(ii) $P_{*}(D)$ is a lower semicontinuous function.

(iii) $P_{*}(D)$ is a viscosity supersolution of (1.1).

Furthermore, assertions (i)-(iii) imply

$$
\Phi(D) \leq P_{*}(D)
$$

so $\Phi=P_{*}$. Thus, the unique classical solution of the differential equation (1.1) with linear growth at infinity is in fact the minimal equilibrium price.

Proof The assertion $P_{*}(D) \leq \Phi(D)$ is obvious, since $\Phi$ is an equilibrium price and $P_{*}$ is the minimal equilibrium price. We also know $P_{*}(D) \geq I(D)$, where

$$
I(D)= \begin{cases}\frac{D}{\lambda+\kappa_{1}}+\frac{\theta \kappa_{1}}{\lambda\left(\lambda+\kappa_{1}\right)}, & \text { if } D \leq \theta, \\ \frac{D}{\lambda+\kappa_{2}}+\frac{\theta \kappa_{2}}{\lambda\left(\lambda+\kappa_{2}\right)}, & \text { if } D \geq \theta\end{cases}
$$

is the intrinsic value (cf. (2.3) of [1]), since the definition of an equilibrium price (Definition 2.1 of [1]) includes this inequality. Theorem 4.1(b) of [1] shows that $\Phi(D)-I(D) \rightarrow 0$ as $|D| \rightarrow \infty$. This gives (i), since $\Phi(D)-P_{*}(D) \leq \Phi(D)-I(D)$.

Assertions (ii) and (iii) are stated and proved in Theorem 5.1 of [1].

For the final conclusion (1.2), consider the variational problem

$$
\inf _{D \in \mathbb{R}}\left\{P_{*}(D)-\Phi(D)\right\} .
$$

If a minimizing sequence tends to $\pm \infty$ then the minimum value is 0 by (i), and (1.2) is true. If on the other hand a minimizing sequence stays bounded, then the minimum is achieved at some $D_{1}$, by (ii). Since $P_{*}$ is a viscosity supersolution we have

$$
-\max \left\{\kappa_{1}\left(\theta-D_{1}\right), \kappa_{2}\left(\theta-D_{1}\right)\right\} \Phi^{\prime}\left(D_{1}\right)-\frac{1}{2} \sigma^{2} \Phi^{\prime \prime}\left(D_{1}\right)+\lambda P_{*}\left(D_{1}\right)-D_{1} \geq 0 .
$$

It follows since $\Phi$ solves (1.1) that

$$
-\lambda \Phi\left(D_{1}\right)+\lambda P_{*}\left(D_{1}\right) \geq 0 .
$$

Since the discount rate $\lambda$ is positive, we conclude that $P_{*}\left(D_{1}\right)-\Phi\left(D_{1}\right) \geq 0$. Thus $P_{*}(D)-\Phi(D) \geq P_{*}\left(D_{1}\right)-\Phi\left(D_{1}\right) \geq 0$, completing the proof of (1.2).

Acknowledgements We thank Yongchao Zhang for pointing out the error in [1] that is fixed by this note.

\section{References}

1. Chen, X., Kohn, R.V.: Asset price bubbles from heterogeneous beliefs about mean reversion rates. Finance Stoch. 15, 221-241 (2011)

2. Crandall, M.G., Ishii, H., Lions, P.L.: User's guide to viscosity solutions of second order partial differential equations. Bull. Am. Math. Soc. 27, 1-67 (1992) 